\title{
List Exons Assessed
}

National Cancer Institute

\section{Source}

National Cancer Institute. List Exons Assessed. NCI Thesaurus. Code C158907.

A request to enter the exons assessed for mutations in this section of the form. 